\title{
Agent-Based Model and Computing Environment Facilitating the Development of Distributed Computational Intelligence Systems
}

\author{
Aleksander Byrski and Marek Kisiel-Dorohinicki \\ AGH University of Science and Technology, Kraków, Poland \\ \{olekb, doroh\}@agh.edu.pl
}

\begin{abstract}
In the paper a simple formalism is proposed to describe the hierarchy of multi-agent systems, which is particularly suitable for the design of a certain class of distributed computational intelligence systems. The mapping between the formalism and the existing computing environment $A g E$ is also sketched out.
\end{abstract}

\section{Introduction}

Realization of multi-agent systems is usually performed utilizing in parallel: a dedicated formalism for describing its structure and behavior, and then generalpurpose design and implementation methods, such as UML and object-oriented programming to achieve a functional system [1]. Of course using general-purpose design and implementation methods has an unquestionable drawback, which is the need to establish a mapping between the model and its implementation each time a new class of systems (or even a novel approach to modeling) is considered. Obviously dedicated implementation tools help to understand the designed system faster and allow to extend its features easier. There exist some frameworks, which facilitate the construction of agent systems, such as Jade, Aglets, or Grasshopper 2]. Other approaches concentrate on specific aspects of agent systems, like AgentSpeak language for describing BDI agent behavior together with its implementation based on Jason and Moise+ facilitate constructing agents interacting in some organizational structure [3]. However, it is obvious that such frameworks are not equally useful for implementing all kinds of multi-agent systems.

Our interests focus on a specific class of agent systems, which use computational intelligence paradigms - particularly hybrid techniques based on the concept of decentralized evolutionary computation [5]. Several variants of these techniques were considered for different goals and problems (e.g. global, multimodal and multi-criteria optimization), and a variety of systems were developed using general-purpose tools. Both the realized systems and proposed models proved useful for the analysis of these techniques - in testing their efficiency for different kinds of problems or exploring their asymptotic behavior [6]. However, still a complete methodology which would bring together the model and its implementation is lacking. Thus in this paper a simple formalism is proposed to describe the particular class of computing multi-agent systems, which is suitable 
for their realization. It is aimed to describe the structure and behavior of the system, yet it does not provide the means for its more sophisticated analysis. A mapping is established between the proposed formalism and an existing computing environment, which was successfully used in the implementation of the above mentioned decentralized evolutionary computation systems.

The paper begins with the presentation of a formalism which allows for describing the structure and behavior of computing agent-based systems. The model of an evolutionary multi-agent system is given as an illustration in the next section. Finally the mapping between the model and the computing environment $A g E$ is shown and some conclusions are drawn.

\section{Structure and Behavior of a Computing MAS}

Obviously a multi-agent system consists of autonomous agents. Thus the proposed model defines MAS as a set of agents, but also a set of actions to be executed by the agents, and the environment represented by some common data, which may acquired by the agents:

$$
A S \ni a s=\left\langle A g, A c t, q r_{1}, \ldots, q r_{m}\right\rangle
$$

where:

$A g \subset A G$ is the set of agents of as,

Act $\subset A C T$ describes actions that may be performed by the agents of as, $q r_{i} \in Q R_{i}, i=1, \ldots, m$ denote queries providing data (knowledge) available for all agents in the environment.

An agent ag may be described as the following tuple:

$$
A G \ni a g=\left\langle i d, t p, d a t_{1}, \ldots, d a t_{n}\right\rangle
$$

where:

$i d \in I D$ is a unique identifier of an agent1,

$t p \in T P$ denotes the type of an agent, depending on its type, an agent is equipped with specific data and may perform specific actions,

$d a t_{i} \in D A T_{i}, i=1, \ldots, n$ represents problem-dependent data (knowledge) gathered by an agent (solutions, resources, outcomes of the observations etc.)

An agent may provide an environment for a group of other agents, which by themselves constitute a multi-agent system, which is essentially different then the one of the "parent" agent. These nested (multi-agent) subsystems introduce a tree-like structure, which will be further referred as physical hierarchy of agents. In fact, the agents of different subsystems form a structure, which is rather a

${ }^{1}$ For each element of the model its domain, which is a finite set of possible values, is denoted by the same symbolic name in upper case, e.g. $I D$ is the set of all possible agent identifiers. 
collection of trees (a forest), which may be perceived as a single tree with a virtual root node. Inner nodes of this structure may be perceived as aggregates of other agents, but of course it is only a simplification, since these sub-agents constitute a complete multi-agent system according to (1). Particular types of agents are often present at particular levels of physical hierarchy - the structure of allowed agent types in the physical hierarchy is called a logical hierarchy.

To identify an agent which provides the environment for a particular multiagent system a mapping is introduced:

$$
\gamma: A S \rightarrow A G \cup\{\varnothing\}
$$

If a certain agent $a g \in A g$ in $a s=\langle A g, A c t\rangle$ provides the environment for $a s^{*}=\left\langle A g^{*}, A c t^{*}\right\rangle$ the mapping will give $\gamma\left(a s^{*}\right)=a g$, and this agent will be called an aggregate for $a s^{*}$. For a multi-agent system at the top of the physical hierarchy $\gamma(a s)=\varnothing$, since there is no real agent providing an environment for this system (symbol $\varnothing$ may be perceived as denoting a virtual root node of the hierarchy).

Let us introduce subsumption relation $\preceq$ in the set of types $T P$, which is used to state whether one type is the specialization of another:

$$
" \preceq " \subset T P \times T P
$$

Relation $\preceq$ defines a partial order in $T P$ (it is reflexive, transitive, antisymmetric). Since the type of an agent determines its features, it is assumed that an agent with a more specific type is able to provide all data and perform all actions of an agent with a more general type, yet of course the realization of the descendant may be different than the parent.

Further deliberations are impossible without giving even the approximation of the system state space:

$$
\begin{aligned}
X & =2^{A S} \\
A S & =2^{A G} \times 2^{A C T}
\end{aligned}
$$

Actual state space will be constrained by the existence of agent types, and the logical hierarchy of particular system, yet the precise description of the state of the whole system is beyond the scope of this paper.

Agents may perform actions in order to change the state of the system. An action is defined as the following tuple:

$$
A C T \ni a c t=\langle t p, \text { pre, post }\rangle
$$

where:

$t p \in T P$ denotes the type of agents allowed to execute the action (only agents of the type $t p$ and descendant types - according to " $\preceq "$ relation - may perform the action), pre $\in X$ is the state of the system which allows for performing action act, post $\in X \times X$ the relation between the state of the system before and after performing action act, 
This tuple may be perceived as so-called Hoare's triple (precondition, operation, postcondition) used e.g. for contract definition in component-based software (see [7]). The descendant types (according to $\preceq$ relation) must hold the preconditions and postconditions of the parents, yet they may extend these conditions by adding new alternatives in preconditions and conjunctions in postconditions. When mentioned conditions are met, the descendant types are fully substitutable for parent types (in the means of contract substitutability [] ).

The following function family is used by an agent of type tp to choose the action to be executed:

$$
\left\{\omega^{t p}: X \rightarrow \mathcal{M}(A C T)\right\}
$$

The choice of the action is thus based on the current state of the system and is defined in stochastic terms. Usually only small part of the system state will be taken into consideration (e.g. the agent's data or it's aggregate's data). Function $\omega$ allows an agent to choose only the actions with compatible (the same or descendant) type:

$$
\begin{gathered}
\forall a g \in A G: a g=\left\langle i d, t p_{1}, d a t_{1}, \ldots, d a t_{n}\right\rangle, \forall a c t \in A C T: a c t=\left\langle t p_{2}, \text { pre }, \text { post }\right\rangle \\
\omega^{t p}(x)(a c t)>0 \Longleftrightarrow t p_{1} \preceq t p_{2}
\end{gathered}
$$

thus introducing a new type of agents requires adding new function for choosing the actions.

To recapitulate, the relations among agents in the whole system may be described using two distinct hierarchies: physical hierarchy (aggregate agents provide an environment for nested multi-agent (sub)systems) and type hierarchy (each agent is assigned a type, which may be a specialization of another type).

\section{Modeling Evolutionary Multi-Agent Systems}

The idea of EMAS (Evolutionary Multi-Agent System) was proposed as a particular technique of decentralized evolutionary computation [95. A variety of applications of this paradigm were considered from typical optimization problems to hybrid computational intelligence systems [10].

The system consists of individual agents decomposed into several subpopulations (demes). Agents contain (partial) solutions of the given optimization problem. They also contain a non-renewable resource called life energy, which is the base of a distributed selection process. Agents exchange their energy based on the fitness value of their solutions. These which gather more energy have greater chances for reproducing, and those with low energy have greater chances of dying. This energy-based selection is used instead of classical global selection mechanisms, because of the assumed autonomy of agents. Agents may also migrate to another subpopulation if they have enough energy.

To facilitate the realization of EMAS, two types of agents are used:

$$
T P=\{i n d, i s l\}
$$

${ }^{2} \mathcal{M}(A)$ denotes the space of probabilistic measures over the measurable set $A$. 
where ind denotes the type of an individual agent (as described above), and $i s l$ - of an aggregate agent, which introduced to manage subpopulations of individual agents (plays a role of an evolutionary island).

Consequently at the top of the physical structure of EMAS there is a system of evolutionary islands:

$$
a s=\langle A g, \varnothing\rangle \quad \gamma(a s)=\varnothing
$$

where:

$$
A g \ni a g=\langle i d, i s l, N b\rangle
$$

and $N b \subset A g$ is the set of neighboring evolutionary islands, which is used to define the topology of migration.

Every evolutionary island $a g$ provides an environment for the population of individual agents:

$$
\begin{array}{r}
\forall a g \in A g \exists a s^{*}=\left\langle A g^{*},\{\text { migr, get,repr,die }\}, \text { find } A g, \text { findLoc }\right\rangle: \\
\qquad a g=\gamma\left(a s^{*}\right)
\end{array}
$$

and an individual agent is defined as:

$$
A g^{*} \ni a g^{*}=\langle i d, i n d, \text { sol, } e n\rangle
$$

where:

$s o l \in S O L$ is the solution of the problem (usually for optimization problems $\left.S O L \subset \mathbb{R}^{n}, n \in \mathbb{N}\right)$,

en $\in \mathbb{R}^{+}$is the amount of energy gathered by the individual,

find $A g: A G \rightarrow \mathcal{M}(A G)$ is the query that allows to choose the neighboring individual agent (another agent present in the same system),

findLoc: $A G \rightarrow \mathcal{M}(A G)$ is the query that allows to choose the neighboring island (using $N b \subset A g$ ).

The set of actions available for individual agents contains:

- migr - migration of an agent from one to another subpopulation,

- get - transfer of a portion of energy from one to another agent,

- repr - creation of a new agent by two parents,

- die - removing of an agent from the system.

Agents use following function to choose the action they intend to perform:

$$
\omega^{\text {ind }}: X \rightarrow \mathcal{U}(\{\text { migr, get, repr }, \text { die }\})
$$

where $\mathcal{U}(\cdot)$ stands for a uniform random distribution.

Action of energy transfer get performed by $a g_{1}^{*}=\left\langle i d_{1}, i n d\right.$, sol $\left._{1}, e n_{1}\right\rangle \in A g^{*}$, which meets $a g_{2}^{*}=$ find $A g\left(a g_{1}^{*}\right)=\left\langle i d_{2}, i n d\right.$, sol $\left._{2}, e n_{2}\right\rangle \in A g^{*}$ (randomly chosen with uniform distribution from its neighbors) may be defined in the following way:

$$
\text { Act } \ni \text { get }=\langle\text { ind, pre, post }\rangle
$$


where:

$$
\begin{gathered}
\text { pre }=\# A g^{*}>1 \\
\text { post }=\left(\varphi\left(s o l_{1}\right)<\varphi\left(s o l_{2}\right) \Rightarrow e n_{1}^{\prime}=e n_{1}+e_{g e t} \wedge e n_{2}^{\prime}=e n_{2}-e_{g e t}\right) \\
\vee\left(\varphi\left(s o l_{1}\right)>\varphi\left(s o l_{2}\right) \Rightarrow e n_{1}^{\prime}=e n_{1}-e_{g e t} \wedge e n_{2}^{\prime}=e n_{2}+e_{g e t}\right)
\end{gathered}
$$

where: $\varphi: S O L \rightarrow \mathbb{R}$ is the fitness function used for the evaluation of solutions, the precise definition of this function is problem-dependent. $e n_{1}^{\prime}$ denotes the value of $e n_{1}$ after performing the action ${ }^{3}, \underline{A}(B)$ denotes the result of the random choice of the value from the set $B$ with distribution $A$.

This action may be performed by an individual agent, which has at least one neighbor (agent in the same MAS subsystem). Depending on the quality of its solution, a part of energy is transferred from the worse to the better agent.

Action of reproduction repr performed by $a g_{1}^{*}=\left\langle i d_{1}, i n d\right.$, sol $\left._{1}, e n_{1}\right\rangle \in A g^{*}$, which meets $a g_{2}^{*}=\left\langle i d_{2}, i n d, s_{2}, e n_{2}\right\rangle \in A g^{*}$ (randomly chosen with uniform distribution from its neighbors) and produces the offspring agent $a g_{3}^{*}=\left\langle i d_{3}\right.$, ind, $\left.\mathrm{sol}_{3}, e n_{3}\right\rangle \in A g^{*}$ may be defined in the following way:

$$
\text { Act } \ni \text { repr }=\langle\text { ind, pre, post }\rangle
$$

where:

$$
\begin{gathered}
\text { pre }=\# A g^{*}>1 \\
\text { post }=e n_{1}>e_{\text {repr }} \wedge e n_{2}>e_{r e p r} \Rightarrow A g^{* \prime}=A g^{*} \cup\left\{a g_{3}^{*}\right\} \\
\wedge e n_{3}=e_{0} \wedge e n_{1}{ }^{\prime}=e n_{1}-\frac{1}{2} e_{0} \wedge e n_{2}{ }^{\prime}=e n_{2}-\frac{1}{2} e_{0} \wedge \operatorname{sol}_{3}=\underline{\chi}\left(\operatorname{sol}_{1}, \text { sol }_{2}\right)
\end{gathered}
$$

where: $\chi: S O L \times S O L \rightarrow \mathcal{M}(S O L)$ is a variation operator (recombination and mutation), its precise definition is problem-dependent.

This action may be performed by an individual agent with at least one neighbor, both with sufficient energy. They create a new agent based on their solutions.

Action of death die performed by $a g^{*}=\langle i d, i n d$, sol, en $\rangle \in A g^{*}$ may be defined in the following way:

$$
\text { Act } \ni \text { die }=\langle\text { ind, pre, post }\rangle
$$

where:

$$
\begin{gathered}
\text { pre }=(e n=0) \\
\text { post }=\left(A g^{* \prime}=A g^{*} \backslash\left\{a g^{*}\right\}\right)
\end{gathered}
$$

This action may be performed by an individual agent which energy level falls to zero. This agent is removed from the system.

Action of migration migr performed by $a g^{*}=\langle i d$, ind, sol, en $\rangle \in A g_{1}^{*}$ : $\exists a s_{1}^{*}=\left\langle A g_{1}^{*}, A c t_{1}^{*}\right\rangle \wedge \gamma\left(a s_{1}^{*}\right)=a g_{1}$ which migrates from $a g_{1}=\left\langle i d_{1}, i s l, N b_{1}\right\rangle$

${ }^{3}$ Here and later the symbol $a^{\prime}$ will denote the value of $a$ after performing the action (usually $a$ is used in precondition and $a^{\prime}$ only in postcondition). This is similar to old operator used in contract specification in Eiffel programming language [8]. 


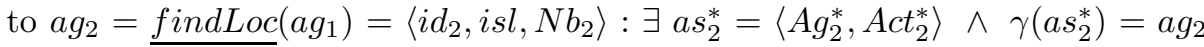
$\left(a g_{2}\right.$ is randomly chosen from all neighbors of $a g_{1}$ with uniform distribution).

$$
\text { Act } \ni \text { migr }=\langle\text { ind,pre,post }\rangle
$$

where:

$$
\begin{gathered}
\text { pre }=a g_{2} \in N b_{1} \\
\text { post }=e n_{1}>e_{\text {migr }} \Rightarrow A g_{1}^{* \prime}=A g_{1}^{*} \backslash\left\{a g_{1}^{*}\right\} \wedge A g_{2}^{* \prime}=A g_{2}^{*} \cup\left\{a g_{1}^{*}\right\}
\end{gathered}
$$

This action may be performed by an individual agent, located in the system with at least two evolutionary islands. The agent with sufficient energy $e_{\text {migr }}$ leaves one island and moves to another.

\section{AgE Computing Environment}

The model presented constitutes a base for the design of the core structure of distributed computing environment $\mathrm{AgE} 4$, developed as an open-source project at Intelligent Information Systems Group of AGH University of Science and Technology. The name reminds that it was primarily dedicated to agent-based evolutionary techniques $(\mathrm{AgE}=$ Agent Evolution), but now it grew up into a general-purpose platform facilitating the implementation of a variety of (not necessarily but most suitably) agent-based simulation and computing systems. Mainstream implementation is realized in Java and follows component approach, which allows for flexible (re)configuration of the system to meet the requirements of particular problems and solving techniques.

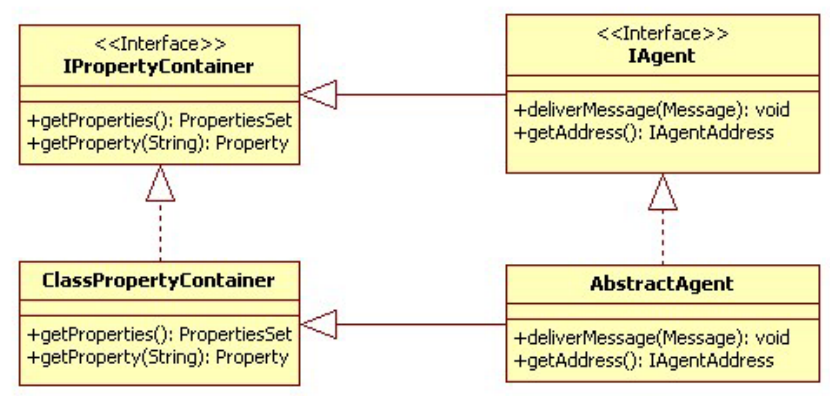

Fig. 1. Properties of objects in AgE computing core

Essential implementation entities that form the computing core of AgE (mainly agents) are realized so as they may be described in terms of properties. A property is a feature of an object, which may be referenced at runtime by its name

\footnotetext{
${ }^{4}$ http://age.iisg.agh.edu.pl/
} 
- to be read, written or even monitored. This functionality is represented by IPropertyContainer interface and may be easily achieved extending an abstract class ClassPropertyContainer (Fig. 1), which provides instrumentation allowing to treat appropriately annotated methods (serving as getters or setters) or fields of a class as its properties.

According to the definition of an agent (2) properties allow for flexible access to agents' data $d a t_{i}$, which facilitates the realization of observation of single agents. It is always possible because a fundamental interface representing an agent IAgent extends IPropertyContainer. Basic agent implementation AbstractAgent extends ClassPropertyContainer to gain this functionality without any additional effort. Also the identification of an agent, which is realized in the form of a unique agent address (AgentAddress class), is provided as a property.

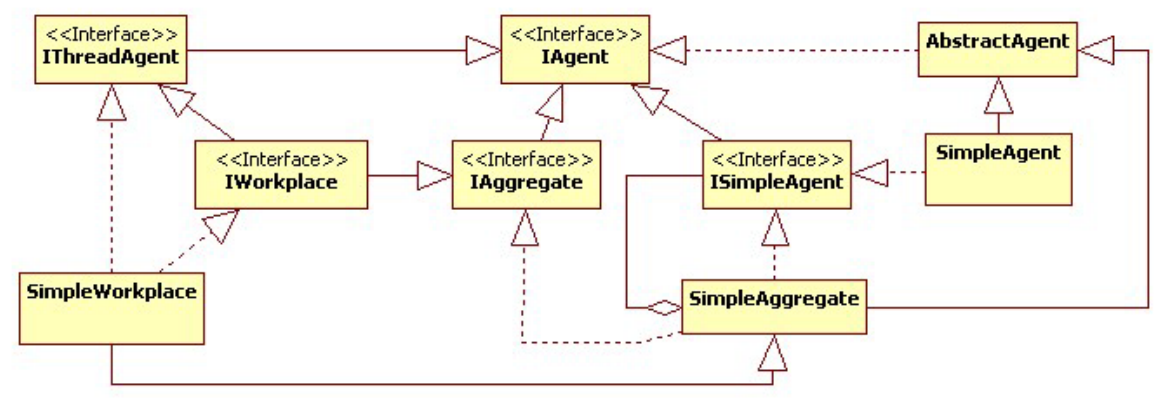

Fig. 2. Different kinds of agent implementations in AgE computing core

Fig. 2 shows different kinds of agent implementations - most notably those, which allow for parallel execution (IThreadAgent), as well as those which work semi-parallelly based on the concept of event-driven simulation (ISimpleAgent) and thus allow for more efficient realization of agent interactions. A specific interface (IAggregate) implies an agent which serves as an environment for the nested multi-agent subsystem. Since IAggregate extends IAgent, the implementation of a tree-like structure of agents introduced in (1) simply follows composite pattern.

The environment for descendant agents is available via dedicated interface IAgentEnvironment (see Fig. 3), which provides identification and both synchronous (queries) and asynchronous (messages) communication facilities. Queries may concern the state of the system an agent is a part of (realized by IQueryable interface) and the state of the "parent" agent's system (query Parent() operation). In case of event-driven execution (as implemented by SimpleAgent class) the environment (ISimpleAgentEnvironment) also provides the mechanism supporting actions (as realized by SimpleAggregate class).

Agents of the top-level multi-agent system (considering the described hierarchy nested subsystems) play special role in the organization of the platform - they work in parallel and may be distributed over the network. They may 


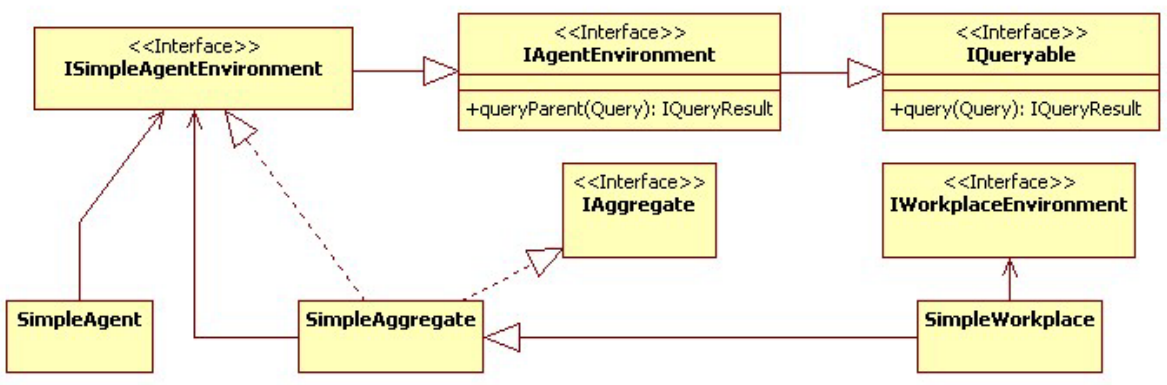

Fig. 3. Environments available in AgE computing core

be understood as roots of local computing environments, because all the agents in one branch of the hierarchy must be executed on one node (a single JVM in Java implementation) — thus they are marked by IWorkplace interface extending IThreadAgent and IAggregate. Obviously there is no special agent providing their environment, but rather there is some infrastructure available via IWorkplaceEnvironment, which provides identification and communication in the possibly distributed way.

\section{Conclusions}

In the paper a simple formal model of a computing multi-agent system was proposed and used to describe a specific computational intelligence system - an evolutionary multi-agent system. The proposed formalism is solely used for design, and that is why such details as the precise definition of the system state space or state transition functions were not taken into consideration. Since several existing EMAS implementations are based on $\mathrm{AgE}$ framework, the mapping between the proposed model and this framework was also shown in the course of the paper.

Further research should allow to extend the model to cover other computation intelligence techniques based on agent paradigm, such as iEMAS (immunological evolutionary multi-agent system) [11] or HGS (hierarchical genetic search) [12. Also the mapping between the proposed formalism and existing models describing these techniques will be provided. Because the implementation of AgE framework continues, the formalism will surely be updated in the near future.

\section{References}

1. Booch, G., Rumbaugh, J., Jacobson, I.: The Unified Modeling Language User Guide. Addison-Wesley, Reading (1998)

2. Trillo, R., Ilarri, S., Mena, E.: Comparison and performance evaluation of mobile agent platforms. In: ICAS 2007: Proceedings of the Third International Conference on Autonomic and Autonomous Systems, Washington, DC, USA. IEEE Computer Society, Los Alamitos (2007) 
3. Bordini, R., Hübner, J., Wooldridge, M.: Programming Multi-Agent Systems in AgentSpeak Using Jason. John Wiley \& Sons, Ltd., Chichester (2007)

4. Hübner, J.F., Sichman, J.S., Boissier, O.: Developing organised multiagent systems using the moise+ model: programming issues at the system and agent levels. Int. J. Agent-Oriented Softw. Eng. 1(3/4), 370-395 (2007)

5. Kisiel-Dorohinicki, M.: Agent-oriented model of simulated evolution. In: Grosky, W.I., Plasil, F. (eds.) SOFSEM 2002. LNCS, vol. 2540, pp. 253-261. Springer, Heidelberg (2002)

6. Byrski, A., Schaefer, R.: Immunological mechanism for asynchronous evolutionary computation boosting. In: ICMAM 2008: European workshop on Intelligent Computational Methods and Applied Mathematics: an international forum for researches, teachers and students, Cracow, Poland (2008)

7. Szyperski, C.: Component Software: Beyond Object-Oriented Programming. Addison-Wesley Longman Publishing Co., Inc., Boston (2002)

8. Meyer, B.: Object-Oriented Software Construction. Prentice Hall PTR, Englewood Cliffs (2000)

9. Cetnarowicz, K., Kisiel-Dorohinicki, M., Nawarecki, E.: The application of evolution process in multi-agent world (MAW) to the prediction system. In: Tokoro, M. (ed.) Proc. of the 2nd Int. Conf. on Multi-Agent Systems (ICMAS 1996). AAAI Press, Menlo Park (1996)

10. Byrski, A., Kisiel-Dorohinicki, M., Nawarecki, E.: Agent-based evolution of neural network architecture. In: Hamza, M. (ed.) Proc. of the IASTED Int. Symp.: Applied Informatics. IASTED/ACTA Press (2002)

11. Byrski, A., Kisiel-Dorohinicki, M.: Immunological selection mechanism in agentbased evolutionary computation. In: Klopotek, M.A., Wierzchon, S.T., Trojanowski, K. (eds.) Intelligent Information Processing and Web Mining: proceedings of the international IIS: IIPWM 2005 conference, Gdansk, Poland. Advances in Soft Computing, pp. 411-415. Springer, Heidelberg (2005)

12. Schaefer, R., Kołodziej, J.: Genetic search reinforced by the population hierarchy. Foundations of Genetic Algorithms 7 (2003) 